\title{
THE SIGNIFICANCE OF YELLOW VERNIX IN THE NEWBORN
}

\author{
BY \\ W. C. TAYLOR, J. A. JAMES and J. L. HENDERSON \\ From the Simpson Maternity Hospital, Edinburgh, and the Department of Child Life and Health, University of \\ Edinburgh
}

(RECEIVED FOR PUBLICATION FEBRUARY 28, 1952)

Yellow staining of the vernix caseosa of the newborn baby is not uncommon. It occurs sometimes in cases of haemolytic disease due to $\mathrm{Rh}$ incompatibility and is a sign of a severely affected infant (Wallerstein, 1948). Otherwise, little attention has been paid to this clinical finding. Clifford (1948) stated that yellow staining of the vernix was observed in about 6 per 1,000 births in the Boston Lying-in Hospital. Of the babies with yellow vernix, $20 \%$ had the icterus gravis type of haemolytic disease with a mortality rate of $36 \%$ and the remaining $80 \%$ of the group had a mortality rate of $6 \%$. He believes that the yellow vernix in the latter group indicates that severe foetal anoxia, probably due to placental disease, has occurred, days or weeks, before delivery. Smith (1951) suggested that the condition was due to inadequate placental function.

The present investigation was carried out in the Simpson Maternity Pavilion, Royal Infirmary, Edinburgh, to determine what factors, other than haemolytic jaundice, cause yellow vernix, and how commonly a poor neonatal prognosis is associated with it.

\section{The Investigation}

All liveborn babies with yellow vernix were seen at birth by a paediatrician. Only those cases in which mustard-yellow vernix was present in the flexures of the limbs as well as over the rest of the body were included. In addition, the skin and nails were stained yellow, and the umbilical cord was brown or green throughout its thickness. Babies in whom there was evidence of $R \mathbf{h}$ incompatibility, shown clinically or by a positive Coombs test or antibodies in the mothers' serum, were excluded, as they constitute a special group with a high mortality and morbidity. The progress of the babies in hospital was observed and a followup study was carried out by questionnaire after discharge. The results obtained were compared with a random series of controls, chosen by select- ing every sixth live birth in the hospital over a short period of time within the period of study. This group of controls is believed to be truly representative of the hospital population during the period of study.

The investigation covered 15 months. During this time 37 babies, including one of twins, were born with yellow vernix, an incidence of approximately 12 per 1,000 hospital births. Seventy babies were chosen as controls. The possible occurrence of yellow vernix in stillborn infants was not investigated.

The following significant results have emerged from the study.

\section{Maternal Factors}

Age of Mother (Table 1, Fig. 1). Fourteen $(37.9 \%)$ of the mothers of affected babies were

TABLE 1

AGE OF MOTHER

\begin{tabular}{c|c|c}
\hline Maternal Age in Years & Series & Controls \\
\hline Under 20 & 0 & $4(6 \%)$ \\
$20-24$ & $6(16 \cdot 3 \%)$ & $24(35 \cdot 8 \%)$ \\
$25-29$ & $13(35 \cdot 1 \%)$ & $13(19.4 \%)$ \\
$30-34$ & $4(10 \cdot 8 \%)$ & $14(20 \cdot 9 \%)$ \\
$35-39$ & $13(35 \cdot 1 \%)$ & $9(13.4 \%)$ \\
40 and over & $1(2 \cdot 7 \%)$ & $3(4 \cdot 5 \%)$ \\
\hline Total & $37(100 \%)$ & $67(100 \%)$ \\
\hline
\end{tabular}

over 35 years of age compared with $12(17 \cdot 8 \%)$ of the mothers in the control group.

Previous Obstetric History (Table 2). Analysis of the obstetric history showed the incidence of

TABLE 2

Previous Obstetric History

\begin{tabular}{|c|c|c|}
\hline & Series & Controls \\
\hline 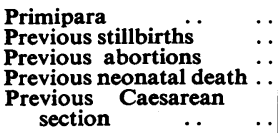 & $\begin{array}{c}18(48 \cdot 6 \%) \\
6(16 \cdot 3 \%) \\
5(13 \cdot 5 \%) \\
2(5 \cdot 4 \%) \\
5(13 \cdot 5 \%)\end{array}$ & $\begin{array}{rr}37 & (55 \cdot 2 \%) \\
3 & (4 \cdot 5 \%) \\
4 & (5 \cdot 0 \%) \\
5 & (7 \cdot 4 \%) \\
3 & (4 \cdot 5 \%)\end{array}$ \\
\hline
\end{tabular}




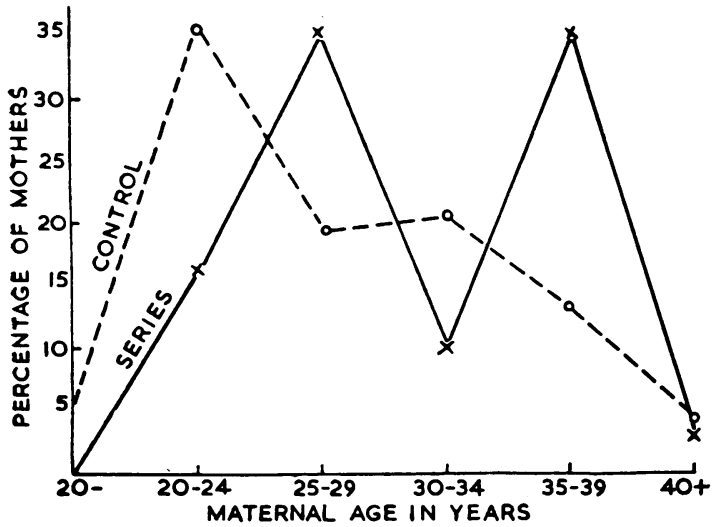

Fig. 1.-Graph to show percentage of mothers in each age group.

previous abortions and stillbirths to be $29.8 \%$ in the mothers of affected babies and only $10.5 \%$ in the mothers in the control groups. Five of the mothers of affected babies had had a previous Caesarean section. In two cases this was carried out for pre-eclamptic toxaemia and in both the infant died early in the neonatal period.

Duration of Pregnancy (Table 3). This was calculated in days from the first day of the last

TABLE 3

\begin{tabular}{|c|c|c|}
\hline Days & Series & Controls \\
\hline $\begin{array}{l}\text { Less than } 250 \\
250-259 \\
260-269 \\
270-279 \\
280-289 \\
290-299 \\
300-309 \\
310 \text { and over }\end{array}$ & $\begin{aligned} & 0 \\
& 0 \\
& 1(3 \cdot 0 \%) \\
& 3(9 \cdot 1 \%) \\
& 9(27 \cdot 3 \%) \\
& 10(30 \cdot 3 \%) \\
& 6(18 \cdot 2 \%) \\
& 4(12 \cdot 1 \%)\end{aligned}$ & $\begin{array}{c}1(1 \cdot 7 \%) \\
4(6.8 \%) \\
10(17.0 \%) \\
15(25 \cdot 4 \%) \\
15(25.4 \%) \\
11(18.6 \%) \\
3(5 \cdot 1 \%) \\
0-\end{array}$ \\
\hline Total & $33(100 \%)$ & $59(100 \%)$ \\
\hline $\begin{array}{l}\text { Total } 280 \text { days } \\
\text { Excluded } \ldots\end{array}$ & $29(87 \cdot 9 \%)$ & $29(49 \cdot 1 \%)$ \\
\hline
\end{tabular}

menstrual period. Twin pregnancies, and cases in which the mother was uncertain of the date, were excluded. The period of gestation in $87.9 \%$ of the mothers of yellow vernix babies lasted more than 280 days, and was in striking contrast to the corresponding figure of $49.1 \%$ in the control group.

Complications of Labour. The incidence of instrumental and operative deliveries in the affected series was $47 \cdot 2 \%$ compared with $15.6 \%$ in the control series. In five $(13.5 \%)$ of the affected cases instrumental delivery was performed because of signs of foetal distress.

Duration of Labour. The average duration of labour in the affected series was $\mathbf{1 8}$ hours and in the control series 13 hours.
The Placenta. This was weighed and examined macroscopically and the length and site of insertion of the cord noted. From this rough examination there did not appear to be any difference between the two groups. In one twin pregnancy, however, one infant had yellow vernix and the other was normal. The normal infant weighed $7 \mathrm{lb} .3 \mathrm{oz}$., and the placenta weighed $24 \mathrm{oz}$. and was of normal appearance. That of the twin with yellow vernix, who weighed $5 \mathrm{lb} .3 \mathrm{oz}$., showed areas of calcification and infarction and weighed only $15 \mathrm{oz}$.

\section{Foetal and Neonatal Factors}

Intra-uterine Foetal Distress. This was diagnosed by undue fluctuation of the foetal heart rate or the staining of the liquor with meconium. It occurred in five $(13.5 \%)$ babies with yellow vernix compared with two $(2.9 \%)$ babies in the control series.

Condition at Birth (Table 4). The infants' condition at birth was assessed as poor when active

TABLE 4

Condition at Birth, Birth Weight, Sex Incidence, Neonatal CONDITION

\begin{tabular}{|c|c|c|c|}
\hline & & Series & Controls \\
\hline \multirow{2}{*}{ Condition at birth } & Good & $24(64 \cdot 8 \%)$ & $61(87 \cdot 1 \%)$ \\
\hline & Poor & $13(35 \cdot 2 \%)$ & $9(12 \cdot 9 \%)$ \\
\hline $\begin{array}{l}\text { Average birth weight } \\
\text { (lb. oz.) }\end{array}$ & & $78 \frac{1}{2}$ & $71 \frac{1}{2}$ \\
\hline \multirow{2}{*}{ Sex incidence } & Male & $24(64 \cdot 8 \%)$ & $41(58 \cdot 5 \%)$ \\
\hline & Female & $13(35 \cdot 2 \%)$ & $29(41 \cdot 5 \%)$ \\
\hline \multirow{3}{*}{ Neonatal condition } & Normal & $31(83 \cdot 8 \%)$ & $66(94 \cdot 3 \%)$ \\
\hline & $\begin{array}{l}\text { Abnor- } \\
\text { mal (in- } \\
\text { cluding } \\
\text { death) }\end{array}$ & $6(16.2 \%)$ & $4(5 \cdot 7 \%)$ \\
\hline & Deaths & $2(5.4 \%)$ & -- \\
\hline
\end{tabular}

resuscitation had to be carried out to aid the establishment of respiration. This was necessary in $35.2 \%$ of the affected infants and in only $12.9 \%$ of the controls.

Birth Weight (Table 4). The affected babies were on the average heavier than the controls $(7 \mathrm{lb}$. $8 \frac{1}{2}$ oz. and $7 \mathrm{lb}$. $1 \frac{1}{2} \mathrm{oz}$. respectively). This is in accordance with the longer average duration of pregnancy already noted in this group.

The Neonatal Condition (Table 4). Two neonatal deaths occurred among the affected infants. One baby had a meningo-myelocoele and died a few days after birth. The other developed acute respiratory distress and collapse on the first day of life and died within 48 hours: post-mortem examination showed evidence of aspiration of 
liquor with hyaline membrane and early bronchopneumonia affecting both lungs.

Two babies in the affected series developed cyanotic attacks relieved by oxygen in the first few days of life; another developed pneumonia, confirmed radiologically, and recovered. One baby developed vomiting and signs of cerebral irritation. None of the control babies died, but four had mild cyanotic attacks in the first few days of life. No other morbidity was observed in either group over a minimum follow-up period of six weeks.

\section{Discussion}

It is probable that yellow staining of the vernix is due to meconium passed at some time during intra-uterine life, although it is not possible to estimate how long before birth this takes place. The fact that the vernix and cord are stained throughout their thickness suggests that it may occur days, or even weeks, before the onset of labour, and is thus quite different from the superficial staining of the vernix which is sometimes seen when intrapartum foetal distress has occurred. Tests for bile in the liquor amnii, however, have been inconclusive. Bevis (1950) was unable to demonstrate bile pigment in cases of erythroblastosis although Gairdner, Lawrie and Hutcheon (1950) found that bile pigment could be present in the liquor amnii in the presence or absence of haemolytic disease.

Clifford (1948) suggested that anoxia might stimulate the passage of meconium during intrauterine life, and that placental degeneration, which was found in a high percentage of his cases of yellow vernix, was the likely cause of the foetal anoxia. Fleming (1943) has shown that the proportion of babies weighing over $8 \mathrm{lb}$. was higher in a group of patients with excessively calcified placentae than in groups with moderate or no calcification, and that the percentage of premature labours was greatest in a group without any calcification of the placenta. This suggests that a relationship exists between the maturity of the foetus and placental degeneration. The results of the present investigation show that there is, on the average, a prolonged gestation time and a higher birth weight than normal in babies born with yellow vernix. They are, therefore, in agreement with Clifford's (1948) view that yellow vernix can be a sign of post-maturity of the foetus. Unfortunately, detailed examination of the placenta was not carried out in the present study, but the case of the twin pregnancy described suggests an association between yellow vernix and degeneration of the placenta.

In the present investigation, as in Clifford's (1948), the incidence of neonatal mortality and morbidity was higher in babies with yellow vernix than in controls, but the numbers are too small to draw definite conclusions of prognostic value. Adverse maternal or placental factors may be partly responsible for the poor prognosis, but the high incidence of abnormal delivery resulting from an abnormally high birth weight may also be important.

Clearly, it is important to recognize that yellow vernix in the newborn is more often associated with placental degeneration than with haemolytic disease. It is necessary to make a correct diagnosis to avoid confusion in the management of such cases.

\section{Summary}

A series of 37 newborn infants with yellow vernix caseosa, not associated with haemolytic disease, has been studied in conjunction with a control series.

Significant maternal factors in the series were: (1) greater maternal age ; (2) prolonged gestation period, with abnormally high birth weight ; (3) increased incidence of abnormal delivery.

Significant foetal and neonatal factors were : (1) an increased incidence of intra-uterine foetal distress; (2) an increased incidence of neonatal apnoea; (3) increased neonatal mortality and morbidity, though the numbers were too small for much significance to be attached to these observations.

It is believed that placental degeneration associated with post-maturity may, by producing foetal anoxia, be the cause of the occurrence of this type of yellow vernix in the newborn.

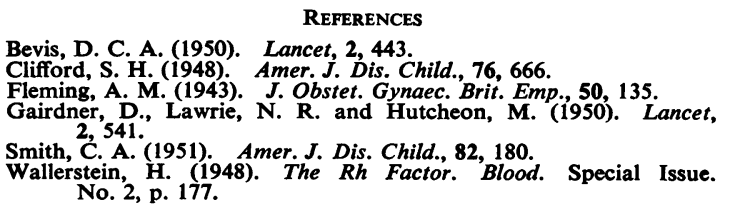

